\title{
035 WHY THE LONG FACE?
}

Aisling Carr, ${ }^{1}$ AL Pelayo, ${ }^{1}$ CJ Whelan, ${ }^{2}$ AD Wechaleka, ${ }^{2}$ JD Gilmore, ${ }^{2}$ PN Hawkins, ${ }^{2}$ MM Reilly ${ }^{1}{ }^{1} U C L ;{ }^{2} N A C$.

\subsection{6/jnnp-2014-309236.35}

We report a 63 year old lady with decreased visual acuity, no wrinkles and difficulty sucking drinks through a straw. Neurological examination revealed bilateral lower motor neuron facial weakness. There was normal tone and power in the limbs and pinprick, temperature and proprioception were intact but vibration sensation was absent to the level of the sternum. Gait was normal but there was mild clumsiness of the hands. No prior family history of neurological disease was known. Electrophysiology revealed normal sensory action potentials.

Ophthalmological examination prompted the diagnosis of hereditary gelsolin amyloidosis which is recognized by the triad of corneal lattice dystrophy, progressive cranial neuropathy and cutis laxa. This was confirmed on finding a heterozygous D241N Gelsolin mutation. The unusual pattern of dissociated vibration and proprioceptive loss has been described in this condition with histopathological amyloid deposition providing eloquent demonstration of the involved anatomy. We present this rare case as an interesting and educational lesson in spinal cord anatomy. 